Classification

Physics Abstracts

$66.70-75.40$

\title{
Magnetic excitations of a Heisenberg spin glass : evidence for a gap in high magnetic fields
}

\author{
C. Marcenat, A. Benoît, A. Briggs \\ Centre de Recherches sur les Très Basses Températures, 166X, 38042 Grenoble Cedex, France
}

C. Arzoumanian, A. M. De Goer

Centre d'Etudes Nucléaires, SBT/Laboratoire de Cryophysique, 85X, 38041 Grenoble Cedex, France

and F. Holtzberg

I.B.M. Research Center, Yorktown-Heights, PO Box 218, New York 10598, U.S.A.

(Reçu le 7 mars 1985, accepté sous forme définitive le 26 avril 1985)

\begin{abstract}
Résumé. - Nous avons effectué des mesures de chaleur spécifique $C(T)$ sur un monocristal de verre de spin isolant $\mathrm{Eu}_{0,44} \mathrm{Sr}_{0,56} \mathrm{~S}$ entre 0,7 et $10 \mathrm{~K}$, et sous champ magnétique $(H=0,4,6$ et 7 T). L'application d'un champ magnétique fort modifie profondément le comportement de $C(T)$, avec une très forte diminution à basse température : la variation quasi linéaire observée en champ nul est remplacée par une variation exponentielle caractéristique de rapparition d'une bande interdite dans le spectre des excitations. Ces résultats, ainsi que ceux de magnétorésistance thermique obtenus antérieurement, peuvent être décrits en supposant l'existence de deux types d'excitations magnétiques : (i) des excitations collectives de très basse énergie et (ii) des excitations à un spin d'ions $\mathrm{Eu}^{2+}$ soumis au champ magnétique extérieur augmenté d'un champ interne égal à 3,2 $\mathrm{T}$ dont nous discutons l'origine.
\end{abstract}

\begin{abstract}
Measurements of the specific heat $C(T)$ of a spin glass $\mathrm{Eu}_{0.44} \mathrm{Sr}_{0.56} \mathrm{~S}$ single crystal have been performed between 0.7 and $10 \mathrm{~K}$, and with applied magnetic fields $(H=0,4,6$ and $7 \mathrm{~T})$. The application of a large magnetic field strongly modifies the $C(T)$ behaviour, with a large decrease at low temperatures : the quasilinear variation observed in zero field is replaced by an exponential variation characteristic of the opening of a gap in the excitation spectrum. These results, together with those of magneto-thermal resistivity obtained previously, can be described with the assumption of two kinds of magnetic excitations : (i) very low energy collective excitations and (ii) single spin excitations of $\mathrm{Eu}^{2+}$ ions in the external magnetic field augmented by some internal field of $3.2 \mathrm{~T}$, the origin of which is discussed.
\end{abstract}

\section{Introduction.}

The system $\mathrm{Eu}_{x} \mathrm{Sr}_{1-x} \mathrm{~S}$ is known to be an example of insulating crystalline material which, in a concentration range $0.13<x<0.65$, displays a magnetic behaviour similar to that found in metallic spin-glasses [1]. The long range RKKY interaction present in these is replaced in $\mathrm{Eu}_{x} \mathrm{Sr}_{1-x} \mathrm{~S}$ by short-range isotropic exchange interactions, which are ferromagnetic between nearest neighbours and antiferromagnetic between next-nearest neighbours of $\mathrm{Eu}^{2+}$ ions. 
The advantage of such a system for studying the low energy excitations is the absence of both structural disorder and conduction electrons : the low temperature thermal properties (specific heat and thermal conductivity) are directly related to characteristic properties of the spin-glass state.

Specific heat measurements have given evidence of a linear contribution which decreases with applied magnetic field but the field range was limited to $1 \mathrm{~T} \mathrm{[2].} \mathrm{On} \mathrm{the} \mathrm{other} \mathrm{hand,} \mathrm{we} \mathrm{have}$ studied the magneto-thermal conductivity up to $7 \mathrm{~T}$ on single crystals $(x=0.44, x=0.017)$ down to $60 \mathrm{mK}$. We have observed an effect specific to the spin-glass sample $x=0.44$, that is an increase of the thermal conductivity with applied magnetic field below $15 \mathrm{~K}$ [3]. We have interpreted this effect as due to resonant phonon scattering by the low energy magnetic excitations responsible for the linear term of the specific heat. These excitations were phenomenologically described as « magnetic TLS » with a constant density of states $n_{0}$. Similar behaviour has been observed on spin-glass samples with different europium concentrations by other authors, who have suggested that an additional path for heat transport " spin-wave like » appears with applied fields [4]. To obtain more information on the magnetic excitations in high fields, we have performed specific heat measurements between $0.7 \mathrm{~K}$ and $10 \mathrm{~K}$ up to $7 \mathrm{~T}$, on the same $x=0.44$ sample used in the previous thermal conductivity work [3]. In this work, the magnetic fields used are far above the critical transition line $H_{\mathrm{c}}(T)$, where the spin-glass phase disappears, as determined by Rajchenbach and Bontemps [5] in the vicinity of $T_{\mathrm{g}}$. This is still true at the lowest temperatures from magnetization measurements that we have performed [6].

\section{Experiments and results.}

The single crystal $\mathrm{Eu}_{0.44} \mathrm{Sr}_{0.56} \mathrm{~S}$ is a parallelepiped with cross section of $\sim 1 \mathrm{~mm}^{2}$ and length $5 \mathrm{~mm}$. The freezing temperature $T_{\mathrm{g}}$ obtained by ac susceptibility at $17 \mathrm{~Hz}$ is $1.8 \mathrm{~K}$ [7]. The specific heat has been measured using a « heat leak » method [8] which can easily be piloted by a microcomputer. The $20.88 \mathrm{mg}$ sample $\left(C \simeq 0.2 \mathrm{~mJ} \mathrm{~K}^{-1}\right.$ at $\left.4 \mathrm{~K}\right)$ was stuck with vacuum grease to a thin sapphire plate on which a heater and a thermometer had been evaporated $\left(C_{\text {add }} \simeq 1.3 \mu \mathrm{J} \mathrm{K} \mathrm{K}^{-1}\right.$ at $4 \mathrm{~K}$ ). This thermometer was calibrated at several fields against ${ }^{3} \mathrm{He}$ and ${ }^{4} \mathrm{He}$ vapour pressure thermometers. Extrapolations to $10 \mathrm{~K}$ were made using a calibrated germanium thermometer. The uncertainty in the specific heat is estimated to be about $5 \%$. The geometry was different from that of the thermal conductivity experiments as the applied magnetic field $H_{\mathrm{a}}$ was perpendicular instead of parallel to the largest dimension of the sample. In this inconvenient but unavoidable geometry, the large demagnetization field excluded a study of the specific heat in low fields.

The results of the specific heat measurements are displayed in figure 1 as a function of temperature, for different values of the magnetic field $\left(H_{\mathrm{a}}=0,4,6\right.$ and $\left.7 \mathrm{~T}\right)$. The zero-field curve is typical

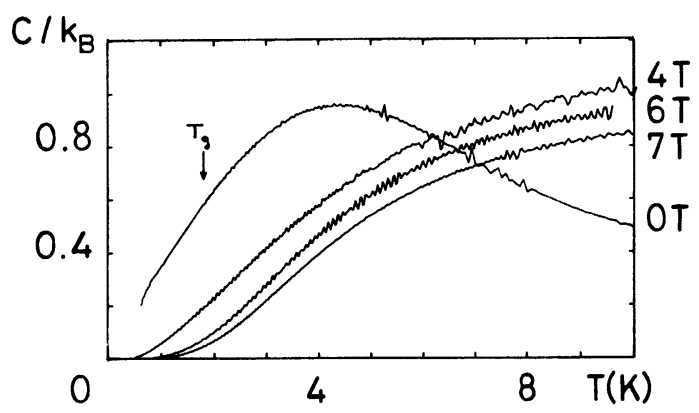

Fig. 1. - Specific heat per Eu atom divided by $k_{\mathrm{B}}$ versus temperature $T$ in various applied fields $H_{\mathrm{a}}(0,4,6$, $7 \mathrm{~T})$ for $\mathrm{Eu}_{0.44} \mathrm{Sr}_{0.56} \mathrm{~S}$. 
of spin glasses : there is a broad maximum well above $T_{\mathrm{g}}\left(T_{\max } \simeq 2.4 T_{\mathrm{g}}\right)$ and no anomaly at $T_{\mathrm{g}}$; in the low temperature range, a roughly linear temperature dependence is observed. The effect of the magnetic field is to strongly decrease the specific heat at low temperatures and to shift the maximum to higher temperatures so that the curves cross the zero field curve. The maxima with applied fields are not observed because of the limited temperature range $(T \leqslant 10 \mathrm{~K})$. At low temperatures, the variation is clearly not linear and is in fact nearly exponential, as we shall discuss later. The decrease of the specific heat with magnetic field is shown more clearly in figure 2 where the ratio $C(H) / C(0)$ is plotted as a function of field at constant temperatures. In this figure, the intensity $H_{\mathrm{d}}$ of the demagnetization field has been taken into account $\left(H=H_{\mathrm{a}}-H_{\mathrm{d}}\right)$; $H_{\mathrm{d}}$ has been obtained from previous measurements of magnetization and demagnetization coefficients for different geometries [6]. For the fields used and below $5 \mathrm{~K}, H_{\mathrm{d}}$ is nearly constant within $3 \%$ and equal to $0.4 \mathrm{~T}$.

The previous thermal conductivity measurements have shown a decrease of the thermal resistivity $W=1 / K$ with applied magnetic field, as illustrated in figure 3 . For the geometry used in this experiment, the demagnetization field $H_{\mathrm{d}}$ is quite small $\left(H_{\mathrm{d}}=0.046 \mathrm{~T}\right.$ within $5 \%$ [6]).

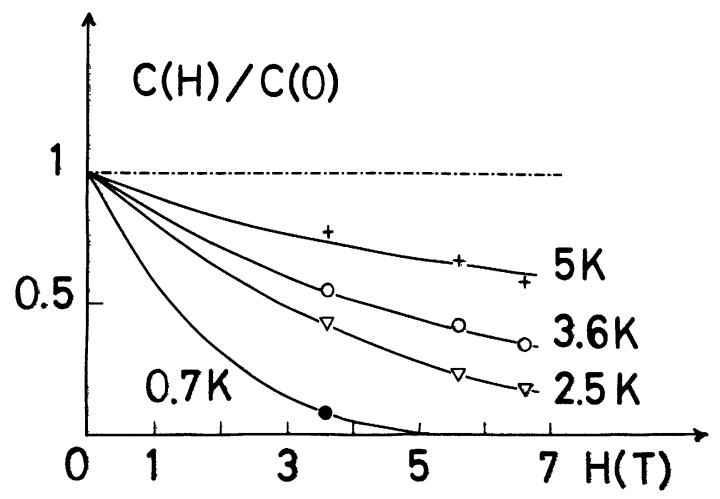

Fig. 2. - Field dependence of the specific heat for various temperatures.

\section{Discussion.}

The measured specific heat is almost completely magnetic in origin. We have estimated the lattice part using a Debye temperature $\theta_{\mathrm{D}}=226 \mathrm{~K}$ [3]. This contribution is less than $1 \%$ of the total specific heat below $6 \mathrm{~K}$ and reaches only $10 \%$ at $10 \mathrm{~K}$. The nuclear specific heat is completely negligeable in our whole temperature range, its relative contribution being less than $10^{-3}$ at $0.7 \mathrm{~K}$. The similarity of the shapes of the curves $C(H) / C(0)($ Fig. 2) and $W(H) / W(0)($ Fig. 3) is therefore qualitatively significant (as both $C$ and $W$ are related to the density of states $n_{0}$ in the previous phenomenological approach [3]) and confirms that the low energy magnetic excitations scatter phonons.

The experimental results of the specific heat are shown again in figure 4 after being corrected for the small lattice contribution. In zero field and below $1 \mathrm{~K}$, the nearly linear behaviour corresponds to $\gamma / k_{\mathrm{B}}=0.37 \mathrm{~K}^{-1} \pm 0.03 \mathrm{~K}^{-1}$, a value smaller than that obtained by Meschede $e$ al . [2] $\left(\gamma / k_{\mathrm{B}}=0.5 \mathrm{~K}^{-1}\right.$ for a $x=0.4$ sample). Our experimental results are in good agreement with the numerical calculations by Krey [9] interpolated for our concentration $x=0.44$, up to $1.5 \mathrm{~K}$, as illustrated in figure 4 (other calculations [10] agree with Krey's results within $10 \%$ ). We have calculated the magnetic entropy from the experimental data and a linear extrapolation below $0.7 \mathrm{~K}$. The entropy at $T_{\mathrm{g}}$ is found to be only $\simeq 25 \%$ of the total magnetic entropy, a result 


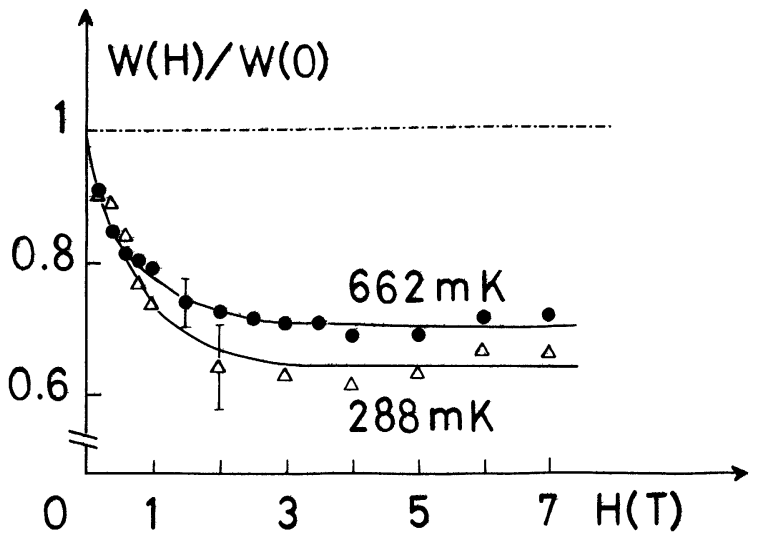

a)

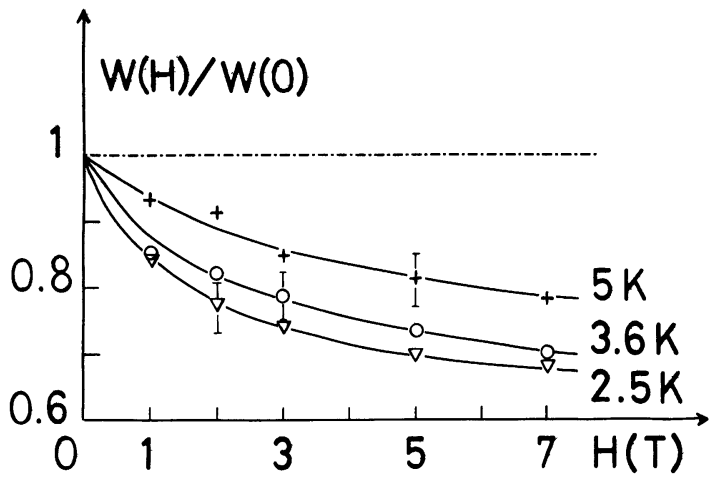

b)

Fig. 3. - Field dependence of the thermal resistivity for various temperatures obtained with different apparatus : $T<1 \mathrm{~K}$ (a) and $T>1 \mathrm{~K}$ (b).

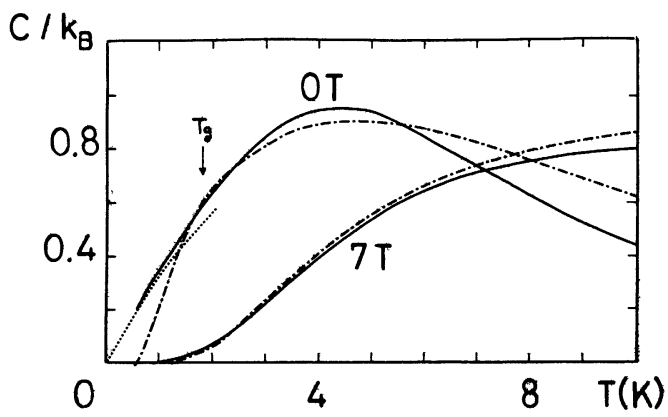

Fig. 4. - Temperature dependence of the magnetic specific heat in zero-field and in $H_{\mathrm{a}}=7 \mathrm{~T}(\longrightarrow)$; calculated Schottky curve for 0 and $10 \mathrm{~T}(-\cdot)$; zero-field specific heat calculated by U. Krey [9] (...) (see text).

similar to those obtained for metallic spin glasses (Au-Fe : $20 \%$ [11], Cu-Mn : $30 \%$ [12]). On the other hand, nearly $90 \%$ of the total magnetic entropy is reached at $10 \mathrm{~K}$, the upper limit of our temperature range. 
Now the very low energy magnetic excitations are drastically reduced by the application of a magnetic field, as noted above. A relatively satisfactory description of the specific heat data with $H_{\mathrm{a}}=7 \mathrm{~T}$ is obtained with a simple Schottky anomaly calculated for the $\mathrm{Eu}^{2+}$ ions with $S=7 / 2$. Such a fit is shown in figure 4 , but it is necessary to use an apparent magnetic field $H^{*}=10 \mathrm{~T}$ instead of the true local magnetic field $H_{\mathrm{i}}=6.8 \mathrm{~T}$. Therefore the system behaves as a paramagnet in high fields, but with a large « internal field » $H_{\mathrm{i}}=3.2 \mathrm{~T}$. With this internal field, we have calculated the Schottky anomalies for the two other values of $H_{\mathrm{a}}$ (not shown in Fig. 4 in view of clarity). The agreement with experiment is less satisfactory when $H_{\mathrm{a}}$ decreases but still good for $H_{\mathrm{a}}=6 \mathrm{~T}\left(H^{*}=9 \mathrm{~T}\right)$. In zero field, the Schottky calculation evidently fails to describe the low temperature part of the data, but gives the right order of magnitude of the specific heat around the maximum (Fig. 4).

From this analysis, it appears that two types of magnetic excitations are present in zero field : (i) very low energy excitations with a constant density of states and (ii) single spin excitations of $\mathrm{Eu}^{2+}$ ions in a strong local field of 3.2 $\mathrm{T}$. The application of a large magnetic field opens a gap as the very low energy excitations are suppressed, the single spin excitations being shifted without modification of the internal field. The previous analysis of the magneto-thermal conductivity results [3] are in agreement with this scheme. The excitations of the first type were described phenomenologically as " magnetic TLS » which scatter low energy phonons resonantly, by analogy with the properties of structural glasses. This phonon scattering is suppressed by the application of a large magnetic field (Fig. 3). The density of states $n_{0}$ is deduced from the specific heat and the product $n_{0} \bar{M}^{2}$ (where $\bar{M}$ is a coupling constant) already known from the analysis of the magneto-thermal conductivity data [3]. From the present work we obtain $n_{0}=1.2 \times$ $10^{37} \mathrm{erg}^{-1} \mathrm{~cm}^{-3}$ and therefore $\bar{M} \simeq 4.3 \times 10^{-4} \mathrm{eV}$. This coupling constant is very small compared to that of structural glasses $(\bar{M} \simeq 1 \mathrm{eV}[13])$.

From a more physical point of view, these very low energy excitations are probably collective excitations, as Ching et al. [10] have shown that they are largely delocalized. In that case, the effect of a magnetic field could be to open a gap $g \mu_{\mathrm{B}} H$ and not to suppress the excitations, but we cannot test this hypothesis from our experiments : practically it is not possible to distinguish a small contribution from the tail of the large Schottky anomaly due to the excitations of the second type, because of the experimental uncertainty on the small values of the specific heat. Phonon scattering by these " spin-wave like " excitations could arise from a modulation of the exchange energy. As the energy range of these excitations is large and extends up to $\sim 15 \mathrm{~K}$ [9], the phonon scattering is noticeable even if the coupling is small. From dimensionality arguments, the coupling constant is of the order of $\delta J /(\delta r / r)$ and can be estimated from the dependence of $J$ with the volume which is known for EuS [14]. We obtain $\sim 4 \times 10^{-4} \mathrm{eV}$, which is effectively small, and surprisingly close to the value of $\bar{M}$ deduced above from the phenomenological analysis.

On the other hand, phonon scattering by the excitations of the second type is too small to be detected as only phonons with particular energies $g \mu_{\mathrm{B}} H_{\mathrm{i}}, 2 g \mu_{\mathrm{B}} H_{\mathrm{i}}$ etc... can be resonantly scattered. Moreover the eigenstates of the $\mathrm{Eu}^{2+}$ ions are nearly pure spin states so that the spin-lattice coupling is very small.

Finally, we discuss the possible origin of the internal field $H_{\mathrm{i}}=3.2 \mathrm{~T}$. Such a large value could exist in a "ferromagnetic cluster ". A crude estimate from the known exchange constants $J_{1}=$ $0.22 \mathrm{~K}$ with $12 x$ nearest neighbours and $J_{2}=0.11 \mathrm{~K}$ with $6 x$ next nearest neighbours [1] leads to a field at the $\mathrm{Eu}^{2+}$ site of $2.3 \mathrm{~T}$, which is of the right order of magnitude. In zero internal field, the calculated specific heat is too large above the maximum (Fig. 4) : this is an indication that the " internal field " must decrease as the temperature increases, which is quite reasonable as the correlation between spins decreases. The existence of strong spin-correlation well above $T_{\mathrm{g}}$ is known from EPR experiments [15].

We note that we have not discussed the possible influence of dipolar interactions, which are 
known to be large in this system, but which have no important effect on the low energy excitation spectrum [16].

In conclusion, from the analysis of the low temperature properties, two types of magnetic excitations appear to be present in the Heisenberg spin-glass $\mathrm{Eu}_{0.44} \mathrm{Sr}_{0.56} \mathrm{~S}$ : " spin-wave like » low energy excitations which scatter acoustic phonons, and single spin excitations of $\mathrm{Eu}^{2+}$ ions in " ferromagnetic cluster », where a large internal field is present. The effect of a large applied magnetic field is to open a gap in the excitations spectrum.

During the time this work was completed, we have been awared of specific heat measurements on $\mathrm{Eu}_{x} \mathrm{Sr}_{1-x} \mathrm{~S}$ crystals with $x=0.25$ and $x=0.54$ which also give evidence of a gap in the excitations in high magnetic fields [17].

\section{Acknowledgments.}

We are very grateful to Professor R. Maynard for useful discussions and to Dr. S. Von Molnar for providing the bolometer used in these experiments. We thank Dr. K. Matho for useful suggestions and Drs G. Lecomte and H. Von Löhneysen for communicating their results before publication.

One of us (C.M.) thanks St-Gobain-Recherche for its support.

\section{References}

[1] Holtzberg, F., Tholence, J. L., Godfrin, H. and Tournier, R., J. Appl. Phys. 50 (3) (1979) 1717; Maletta, H. and Felsch, W., Phys. Rev. B 20 (1979) 124 ;

Maletta, H. and Felsch, W., Z. Phys. B 37 (1980) 55.

[2] Meschede, D., Steglich, F., Felsch, W., Maletta, H. and Zinn, W., Phys. Rev. Lett. 44 (1980) 102.

[3] Arzoumanian, C., De Goer, A. M., Salce, B. and Holtzberg, F., J. Physique Lett. 44 (1983) L-39. Arzoumanian, C., Salce, B., De Goer, A. M. and Holtzberg, F., in Phonon Scattering in Condensed Matter, eds. : W. Eisenmenger, K. Lassmann and S. Döttinger (Springer-Verlag) 1983, p. 460.

[4] Lecomte, G. V., LöhneYsen, H. V. and ZinN, W., J. Magn. Magn. Mater. 38 (1983) 235.

[5] RajChenbach, J. and Bontemps, N., J. Physique Lett. 44 (1983) L-799.

[6] Arzoumanian, C., Chouteau, G., El Hafidi, M., Tholence, J. L., Note Interne SBT/LCP no 123 (1985);

Arzoumanian, C., Thèse d'Etat, Grenoble (1985).

[7] Holtzberg, F., Francavilla, T. L., Huang, C. Y. and Tholence, J. L., J. Appl. Phys. 53 (3) (1982) 2229.

[8] Marcenat, C. et al., to be published.

[9] KREY, U., Z. Phys. B 38 (1980) 243.

[10] Ching, W. Y., Huber, D. L. and Leung, K. M., Phys. Rev. 21 (1980) 3708.

[11] Wenger, L. E. and Keesom, P. H., Phys. Rev. B 11 (1975) 3497.

[12] Wenger, L. E. and Keesom, P. H., Phys. Rev. B 13 (1976) 4053.

[13] Hunklinger, S. and Arnold, W., in Physical Acoustics, vol. XII, ed. by W. P. Mason and R. N. Thurston (Academic Press) 1976, p. 203.

[14] LÉvy, F., Physik Kondens. Matiere 10 (1969) 71.

[15] Deville, A., Arzoumanian, C., Gaillard, B., Blanchard, D., Jamet, J. P. and Maletta, H., J. Physique 42 (1981) 1641.

[16] KReY, U., J. Magn. Magn. Mater. 28 (1982) 231.

[17] LeCOMte, G. V., private communication.

Commission paritaire $\mathrm{N}^{\circ} \mathbf{5 9 . 0 2 4}$

(C) Les Editions de Physique 1985

Directrice de la Publication : Jeanne BERGER

Imprimé en France. - JOUVE, 18, rue Saint-Denis, 75001 PARIS

No 34114. Dépót légal : Juin 1985 